\title{
ALTERACIONES METABÓLICAS EN NIÑOS CON DEFICIENCIA DE HORMONA DE CRECIMIENTO
}

\section{Metabolic disturbances in children with growth hormone deficiency}

\author{
Francisco Carvajal Martínez ${ }^{\mathrm{a}}$, Yuraimi Piz Ramos ${ }^{\mathrm{b}}$ y Emma Domínguez Alonso ${ }^{c}$
}

Recibido: 26 de noviembre, $2020 \bullet$ Aprobado: 19 de enero, 2021

Cómo citar: Carvajal Martínez F, Piz Ramos Y, Domínguez Alonso E. Alteraciones metabólicas en niños con deficiencia de hormona de crecimiento. cysa [Internet]. 12 de octubre de 2021 [citado 15 de octubre de 2021];5(3):89-100. Disponible en: https://revistas.intec. edu.do/index.php/cisa/article/view/2304

\section{Resumen}

El déficit de hormona del crecimiento (DGH) en la infancia se asocia a factores que aceleran la aterosclerosis.

Objetivo: describir las alteraciones metabólicas en niños con deficiencia de hormona de crecimiento.

Método: se realizó un estudio observacional, descriptivo y transversal que incluyó 53 pacientes con DGH a los cuales se les realizó un interrogatorio, examen físico y los estudios complementarios: prueba de tolerancia a la glucosa oral (PTG-O), lipidograma e insulinemia en ayunas.

Resultados: predominó la hipertrigliceridemia en el sexo femenino, mientras las elevaciones de las LDL fueron más frecuentes en los menores de 5 años. Los valores de tensión arterial (TA), circunferencia de la cintura (CC) y el índice de masa corporal (IMC) se incrementan significativamente con la edad.

Conclusiones: en los niños predominaron las alteraciones corporales, mientras que en las niñas fueron las alteraciones del lipidograma.

Palabras clave: deficiencia de hormona de crecimiento; niños; alteración metabólica, factor de riesgo.

\footnotetext{
a Profesor Titular y Consultante de Endocrinología. Investigador Titular y de Mérito Especialista de 1er. y 2do. Grado en Endocrinología. Departamento de Endocrinología Pediátrica. Instituto Nacional de Endocrinología. (INEN) La Habana, Cuba. ORCID: 0000-0002-8480-1360 Correo-e:endoped@infomed.sld.cu
}

\begin{abstract}
Growth hormone deficiency (DGH) in childhood is associated with promote high cardiovascular morbility.

Objective: To describe the metabolic disturbances in children with growth hormone deficiency.

Method: A study was carried out that included 53 patients with DGH to which an interrogation, physical examination and complementary studie were carried out oral glucose tolerance test (PTG), lipidogram and fasting insulinemia.
\end{abstract}

Results: hypertriglyceridemia predominated in females, while LDL elevations were more frequent in children under 5 years of age. Blood pressure (TA), weist circumference (CC), body mass index (IMC) values increase significantly with age.

Conclusions: In boys, body alteration predominate while in girls was the lipidogram alteratons

Keywords: growth hormone deficiency; children; metabolic disturbance; risk factor.

\footnotetext{
b Especialista de 1er. Grado en Endocrinología y en Medicina General Integral. La Habana, Cuba. ORCID: 0000-0002-4577-4486

${ }^{\mathrm{c}}$ Especialista en Bioestadística Médica, Profesor Auxiliar e Investigador Auxiliar. INEN. La Habana, Cuba. ORCID: 0000-0002-2289-0345
} 


\section{Introducción}

El déficit de hormona de crecimiento (DGH), aislado o asociado a otras deficiencias hormonales, es una afección de naturaleza congénita o adquirida; se caracteriza por la ausencia parcial o total de la hormona de crecimiento $(\mathrm{GH})$ detectable en el plasma. ${ }^{1}$ El DGH tiene un efecto negativo en la composición corporal y el estado metabólico a través de la presencia de otros factores de riesgo conocidos, como: alteraciones en la composición corporal con mayor acumulación de grasa visceral, disminución de la masa magra, dislipidemia, resistencia a la insulina (RI), intolerancia a los hidratos de carbono ${ }^{2,3}$, el aumento de los niveles de lipoproteínas de baja densidad (LDL) y colesterol total $(\mathrm{Ct})$, de los niveles de triglicéridos basales y tras ingesta, y del índice aterogénico. Así como el descenso de los niveles de lipoproteínas de alta densidad (HDL). Existen pocas publicaciones sobre el tema y los resultados difieren entre sí. Ramistella et al., reportan que no hay alteración en el perfil lipídico ${ }^{4}$, mientras otros solo notifican cambios mínimos en los niveles de lípidos ${ }^{5,6}$ en niños con DGH sin tratamiento. Otros estudios demostraron elevación del Ct y LDL al compararlo con un grupo control sin DGH, mientras que el HDL y los triglicéridos eran similares entre ambos grupos. ${ }^{5,7,8}$

El tratamiento con rhGH en niños conlleva una composición corporal más favorable con menor porcentaje de masa grasa y mayor de masa magra. Tiene un efecto positivo sobre el perfil lipídico, ya que se observa una reducción significativa en el índice aterogénico. Además, los niveles de $\mathrm{Ct}$ y de LDL descienden ya desde el primer año de tratamiento, permaneciendo en rango normal posteriormente. ${ }^{9}$ Ejerce, además, un efecto beneficioso sobre la tensión arterial que se mantiene una vez suspendido el tratamiento. ${ }^{9}$

\section{Método}

El estudio se desarrolló en el departamento de Pediatría del Instituto Nacional de Endocrinología
(INEN), La Habana, desde diciembre 2018 hasta mayo del 2019. Estuvo constituido por el total de los nińos y adolescentes $(n=53)$ con diagnóstico de DGH que acudieron a nuestra consulta, en el período evaluado, con edades comprendidas entre 2 y 19 ańos y DGH aislado idiopático comprobado en nuestro servicio mediante Test de sensibilidad a la insulina o Test de Clonidina. Se excluyeron aquellos con enfermedad aguda severa en los 6 meses previos al estudio, evidencia de enfermedad cardiovascular, respiratoria, renal, hepática o endocrina asociada, niños con fenotipos dismórficos como displasia esquelética o síndrome de Turner y crecimiento intrauterino retardado. Se realizó interrogatorio y examen físico. Se calculó el Índice cintura talla (ICT) o de adiposidad abdominal según la fórmula: circunferencia de la cintura/talla. Se estableció los valores mayores de 0.5 como señal de presencia de adiposidad abdominal incrementada. ${ }^{10}$ Para la medición de la presión arterial se siguieron las instrucciones de la Guia cubana para la prevención, diagnóstico y tratamiento de la $H T A^{11}$. Se les realizó extracción de sangre para: prueba de tolerancia a la glucosa oral (PTG-O), lipidograma: colesterol, triglicéridos y LDLc y la insulinemia basal.

La valoración de la PTG-O se categorizó según los valores de glucemia ${ }^{12} \mathrm{en}$ : Normal: Glucemia Ayunas $\leq 5.5 \mathrm{mmol} / \mathrm{l}, \mathrm{PTG}-\mathrm{O} 2$ horas $\leq 7.7 \mathrm{mmol} / \mathrm{l}$, Prediabetes: Glucemia en ayunas $5.6-6.9 \mathrm{mmol} / \mathrm{l}$, PTO-O 2 horas 7.8-11.0mmol/l, Diabetes Mellitus: Glucemia en Ayunas $\geq 7.0 \mathrm{mmol} / \mathrm{l}$, PTG-O 2 horas $\geq 11.1 \mathrm{mmol} / \mathrm{l}$.

Utilizando los valores de glucemia e insulinemia en ayunas se calculó el índice de resistencia a la insulina HOMA-IR mediante la fórmula: RI = (insulinemia ayuna $(\mu \mathrm{U} / \mathrm{ml}) \times$ glucemia ayuna $(\mathrm{mmol} / \mathrm{l})$ 122,5 . Se consideró resistencia a la insulina tomando los valores del índice HOMA-IR $\geq 75$ percentil para cada grupo de edad: de $5.1-10$ años $\geq 1.67$; de 10.1 - $15 \geq 2.53$; de $15.1-19 \geq 2.52 .{ }^{13}$ Los valores de normalidad del lipidograma se consideraron según 
las Guias para el manejo de la dislipidemia y prevención de la aterosclerosis, publicadas en el 2017. ${ }^{14}$ El CT es normal $<5,2 \mathrm{mmol} / \mathrm{L}$, LDLc $<3,4 \mathrm{mmol} / \mathrm{L}$ y los $\mathrm{Tg}<1.7 \mathrm{mmol} / \mathrm{L}$, fuera de estos rangos se consideran de riesgo para desarrollar enfermedades cardiovasculares. La presencia de alteración en alguno de los parámetros del lipidograma se definió como dislipoproteinemia. La LDLc se determinó por método enzimático directo.

\section{Resultados}

Se incluyeron en el estudio un total de 53 nińos. La media de edad de la muestra fue de 12,55 $\pm 3,26$ distribuidos en los siguientes grupos de edades:
2: $32(60,4 \%)$ presentan más de trece años, seguido de 19 nińos (35,8\%) de 6 a 12 ańos y solo el 3,8\% menor de 5 años. Las frecuencias de los factores de riesgo distribuidos por sexo se muestran en la tabla 1. Las alteraciones de la TA solo se presentaron en el sexo masculino; afectando 4 pacientes. Pero la RI fue más frecuente en el sexo masculino representando el 46,3\%, mientras en el sexo femenino solo representó el 33,3\%. La presencia de DLP fue estadísticamente significativa ( $\mathrm{p}=0,049)$, afectando a 7 (58\%) del sexo femenino y solo al $27 \%$ de los varones $\mathrm{El}$ sexo femenino presentó los triglicéridos elevados en el $66,7 \%$ y los varones solo en un $22 \%$ $(\mathrm{p}=0,006)$.

Tabla 1. Distribución de los factores de riesgo según sexo

\begin{tabular}{|c|c|c|c|c|c|c|}
\hline Factores de riesgo & Categoría & $M(n=41)$ & $\%$ & $F(n=12)$ & $\%$ & $\mathbf{P}$ \\
\hline \multirow{2}{*}{ Adiposidad abdominal } & Presenta $(n=17)$ & 13 & 31,7 & 4 & 33,3 & \multirow{2}{*}{0,587} \\
\hline & No presenta $(\mathrm{n}=36)$ & 28 & 68,3 & 8 & 66,7 & \\
\hline \multirow{3}{*}{ Tensión Arterial } & Normal $(\mathrm{n}=49)$ & 37 & 90,2 & 12 & 100,0 & \multirow{3}{*}{0,531} \\
\hline & Prehipertensión (n=2) & 2 & 4,9 & 0 & 0,0 & \\
\hline & HTA $(n=2)$ & 2 & 4,9 & 0 & 0,0 & \\
\hline \multirow{5}{*}{ Valoración nutricional } & Bajo Peso $(n=6)$ & 4 & 9,8 & 2 & 16,7 & \multirow{5}{*}{0,301} \\
\hline & Delgado $(\mathrm{n}=5)$ & 4 & 9,8 & 1 & 8,3 & \\
\hline & Normopeso $(\mathrm{n}=27)$ & 22 & 53,7 & 5 & 41,7 & \\
\hline & Sobrepeso $(\mathrm{n}=9)$ & 5 & 12,2 & 4 & 33,3 & \\
\hline & Obeso $(n=6)$ & 6 & 14,6 & 0 & 0,0 & \\
\hline \multirow{3}{*}{$\begin{array}{l}\text { Tolerancia a los hidratos de } \\
\text { carbono }\end{array}$} & Normal $(n=43)$ & 33 & 80,5 & 10 & 83,3 & \multirow{3}{*}{0,859} \\
\hline & Prediabetes $(n=9)$ & 7 & 17,1 & 2 & 16,7 & \\
\hline & $\mathrm{DM}(\mathrm{n}=1)$ & 1 & 2,4 & 0 & 0,0 & \\
\hline \multirow{2}{*}{ Colesterol } & Normal $(n=46)$ & 35 & 85,4 & 11 & 91,7 & \multirow{2}{*}{0,496} \\
\hline & Elevado $(\mathrm{n}=7)$ & 6 & 14,6 & 1 & 8,3 & \\
\hline \multirow{2}{*}{ Triglicéridos } & Normal $(n=36)$ & 32 & 78,0 & 4 & 33,3 & \multirow{2}{*}{0,006} \\
\hline & Elevado $(\mathrm{n}=17)$ & 9 & 22,0 & 8 & 66,7 & \\
\hline \multirow{2}{*}{ LDLc } & Normal $(n=48)$ & 38 & 92,7 & 10 & 83,3 & \multirow{2}{*}{0,315} \\
\hline & Elevado $(\mathrm{n}=5)$ & 3 & 7,3 & 2 & 16,7 & \\
\hline \multirow{2}{*}{ DLP } & Presenta $(\mathrm{n}=18)$ & 11 & 26,8 & 7 & 58,3 & \multirow{2}{*}{0,049} \\
\hline & No presenta $(\mathrm{n}=35)$ & 30 & 73,2 & 5 & 41,7 & \\
\hline \multirow{2}{*}{ Resistencia insulínica } & Presenta $(\mathrm{n}=23)$ & 19 & 46,3 & 4 & 33,3 & \multirow{2}{*}{0,323} \\
\hline & No presenta $(\mathrm{n}=30)$ & 22 & 53,7 & 8 & 66,7 & \\
\hline
\end{tabular}


En la tabla 2 se presentan las diferencias de las medias y la desviación estándar de las variables de tendencia central. En el caso de los valores de insulinemia y el HOMA-IR se emplearon medianas y rango, ya que en estas variables existía gran dispersión de los valores. Se encontró diferencias estadísticamente significativas $(\mathrm{p}=0.000)$ en los valores de TAS; siendo superior en el sexo masculino ( 98,17 vs. 83,75). Igualmente, los valores de las medias de glucemias en ayunas fueron superiores en el sexo masculino (4,87 vs. 4,29$)$ siendo estadísticamente significativo $(\mathrm{p}=0,047)$. Los valores medios de los triglicéridos fueron superiores en el sexo femenino $(2,07 \mathrm{mmol} / \mathrm{l})$ que en el masculino $(1,43 \mathrm{mmol} / \mathrm{l})$ para un valor de $\mathrm{p}=0,001$.

Tabla 2. Variables de tendencia central según sexo

\begin{tabular}{|l|l|l|l|}
\hline Variable & $\mathbf{M}(\mathbf{n}=\mathbf{4 1})$ & $\mathbf{F}(\mathbf{n}=\mathbf{1 2})$ & $\mathbf{P}$ \\
\hline TAS $(\mathrm{mmHg})$ & $98,17 \pm 11,05$ & $83,75 \pm 9,80$ & 0,000 \\
\hline TAD $(\mathrm{mmHg})$ & $62,93 \pm 8,59$ & $56,67 \pm 12,12$ & 0,047 \\
\hline CC $(\mathrm{cm})$ & $65,59 \pm 11,01$ & $62,00 \pm 11,65$ & 0,269 \\
\hline IMC $\left(\mathrm{Kg} / \mathrm{m}^{2}\right)$ & $19,07 \pm 4,46$ & $17,54 \pm 4,34$ & 0,181 \\
\hline ICT & $0,47 \pm 0,07$ & $0,47 \pm 0,08$ & 0,725 \\
\hline Glucemia Ay $(\mathrm{mmol} / \mathrm{l})$ & $4,86 \pm 0,82$ & $4,29 \pm 0,84$ & 0,047 \\
\hline Glucemia $2 \mathrm{~h}(\mathrm{mmol} / \mathrm{l})$ & $5,55 \pm 1,34$ & $5,39 \pm 1,39$ & 0,509 \\
\hline Colesterol $(\mathrm{mmol} / \mathrm{l})$ & $4,1 \pm 0,89$ & $4,42 \pm 0,70$ & 0,187 \\
\hline Tg $(\mathrm{mmol} / \mathrm{l})$ & $1,43 \pm 1,35$ & $2,07 \pm 0,85$ & 0,001 \\
\hline LDL (mmol/l) & $2,34 \pm 0,71$ & $2,63 \pm 0,78$ & 0,095 \\
\hline Insulinemia & $9,8(55,9)^{*}$ & $9,6(36,2)^{*}$ & 0,742 \\
\hline HOMA-IR & $2,24(14,12)^{*}$ & $1,75(6,06)^{*}$ & 0,339 \\
\hline *Se utilizan medianas y rangos. & & & \\
\hline
\end{tabular}

En la tabla 3 se muestra la distribución de los factores de riesgo según los grupos de edades. Las alteraciones de las LDL resultaron estadísticamente significativas $(\mathrm{p}=0,000)$, están presentes en el 100 $\%$ del grupo entre 2 y 5 ańos, y solo en $3(9,4 \%)$ de los mayores de 13 años. Se presentó pre hipertensión y HTA en los mayores de 13 años con 2 casos $(6,3 \%)$ en cada categoría. El $100 \%$ de los niños entre 2 y 5 años mostraron RI, mientras que en el resto de los grupos representó aproximadamente el $40 \%$. La hipertrigliceridemia se detectó en el $50 \%$ de los niños entre 2 y 5 años. La DLP se reportó en el $100 \%$ de los niños entre 2 y 5 años, y en el $31 \%$ del resto de los grupos ( $\mathrm{p}=0,133)$. 
Tabla 3. Distribución de los factores de riesgo según grupos de edades

\begin{tabular}{|c|c|c|c|c|c|c|c|c|}
\hline $\begin{array}{l}\text { Factores de } \\
\text { riesgo }\end{array}$ & Categoría & $\begin{array}{l}2-5 \\
\text { años } \\
(n=2)\end{array}$ & $\%$ & $\begin{array}{l}6-12 \\
\text { años } \\
(n=19)\end{array}$ & $\%$ & $\begin{array}{l}\geq 13 \\
\text { ańos } \\
(n=32)\end{array}$ & $\%$ & $\mathbf{P}$ \\
\hline \multirow{2}{*}{$\begin{array}{l}\text { Adiposidad abdo- } \\
\text { minal }\end{array}$} & Presenta $(\mathrm{n}=17)$ & 0 & 0,0 & 7 & 36,8 & 10 & 31,3 & \multirow{2}{*}{0,562} \\
\hline & No presenta $(\mathrm{n}=36)$ & 2 & 100,0 & 12 & 63,2 & 22 & 68,8 & \\
\hline \multirow{3}{*}{ Tensión Arterial } & Normal $(n=49)$ & 2 & 100,0 & 19 & 100,0 & 28 & 87,5 & \multirow{3}{*}{0,585} \\
\hline & Prehipertensión $(\mathrm{n}=2)$ & 0 & 0,0 & 0 & 0,0 & 2 & 6,3 & \\
\hline & $\operatorname{HTA}(\mathrm{n}=2)$ & 0 & 0,0 & 0 & 0,0 & 2 & 6,3 & \\
\hline \multirow{5}{*}{$\begin{array}{l}\text { Valoración nutri- } \\
\text { cional }\end{array}$} & Bajo Peso $(n=6)$ & 0 & 0,0 & 2 & 10,5 & 4 & 12,5 & \multirow{5}{*}{0,489} \\
\hline & Delgado $(\mathrm{n}=5)$ & 1 & 50,0 & 0 & 0,0 & 4 & 12,5 & \\
\hline & Normopeso $(n=27)$ & 1 & 50,0 & 12 & 63,2 & 14 & 43,8 & \\
\hline & Sobrepeso $(\mathrm{n}=9)$ & 0 & 0,0 & 3 & 15,8 & 6 & 18,8 & \\
\hline & Obeso $(n=6)$ & 0 & 0,0 & 2 & 10,5 & 4 & 12,5 & \\
\hline \multirow{3}{*}{$\begin{array}{l}\text { Tolerancia a } \\
\text { los hidratos de } \\
\text { carbono }\end{array}$} & Normal $(n=43)$ & 2 & 100,0 & 15 & 78,9 & 26 & 81,3 & \multirow{3}{*}{0,859} \\
\hline & Prediabetes $(\mathrm{n}=9)$ & 0 & 0,0 & 4 & 21,1 & 5 & 15,6 & \\
\hline & $\mathrm{DM}(\mathrm{n}=1)$ & 0 & 0,0 & 0 & 0,0 & 1 & 3,1 & \\
\hline \multirow{2}{*}{ Colesterol } & Normal $(n=46)$ & 2 & 100,0 & 17 & 89,5 & 27 & 84,4 & \multirow{2}{*}{0,746} \\
\hline & Elevado $(\mathrm{n}=7)$ & 0 & 0,0 & 2 & 10,5 & 5 & 15,6 & \\
\hline \multirow{2}{*}{ Triglicéridos } & Normal $(\mathrm{n}=36)$ & 1 & 50,0 & 14 & 73,7 & 21 & 65,6 & \multirow{2}{*}{0,718} \\
\hline & Elevado $(\mathrm{n}=17)$ & 1 & 50,0 & 5 & 26,3 & 11 & 34,4 & \\
\hline \multirow{2}{*}{ LDLc } & Normal $(n=48)$ & 0 & 0,0 & 19 & 100,0 & 29 & 90,6 & \multirow{2}{*}{0,000} \\
\hline & Elevado $(\mathrm{n}=5)$ & 2 & 100,0 & 0 & 0,0 & 3 & 9,4 & \\
\hline \multirow{2}{*}{ DLP } & Presenta $(n=18)$ & 2 & 100,0 & 6 & 31,6 & 10 & 31,3 & \multirow{2}{*}{0,133} \\
\hline & No presenta $(\mathrm{n}=35)$ & 0 & 0,0 & 13 & 68,4 & 22 & 68,8 & \\
\hline \multirow{2}{*}{$\begin{array}{l}\text { Resistencia insu- } \\
\text { línica }\end{array}$} & Presenta $(n=23)$ & 2 & 100,0 & 8 & 42,1 & 13 & 40,6 & \multirow{2}{*}{0,256} \\
\hline & No presenta $(\mathrm{n}=30)$ & 0 & 0,0 & 11 & 57,9 & 19 & 59,4 & \\
\hline
\end{tabular}

Los valores medios de las variables cuantitativas según los grupos de edades se muestran en la tabla 4. La TAS y la TAD presentan diferencias significativas entre los grupos, $\mathrm{p}=0,009$ y $\mathrm{p}=0,005$. Las cifras de las medias de estas variables en los niños entre 2 y 5 años son $75 / 45 \mathrm{~mm} / \mathrm{Hg}$, entre 6 y 12 años de $91 / 58$ $\mathrm{mmHg}$ y en los mayores de 13 años $98 / 64 \mathrm{~mm} / \mathrm{Hg}$.
La CC tuvo valores medios de $50 \mathrm{~cm}$ en niños entre 2 y 5 años incrementándose hasta $68,5 \mathrm{~cm}$ en mayores de 13 ańos, lo que resultó significativo $(\mathrm{p}=0,002)$. De igual manera se comportó el IMC $(\mathrm{p}=0,014)$ donde en el primer grupo fue de $14 \mathrm{Kg} /$ $\mathrm{m}^{2}$, entre 6 y 12 años fue de $17 \mathrm{Kg} / \mathrm{m}^{2}$ y en los mayores de 13 ańos fue de $19,9 \mathrm{Kg} / \mathrm{m}^{2}$. 
Tabla 4. Variables de tendencia central según grupo de edades

\begin{tabular}{|c|c|c|c|c|}
\hline Variables & $\begin{array}{l}2-5 \text { ańos } \\
(n=2)\end{array}$ & $\begin{array}{l}\text { 6-12 años } \\
(n=19)\end{array}$ & $\begin{array}{l}\geq 13 \text { ańos } \\
(n=32)\end{array}$ & $\mathbf{P}$ \\
\hline TAS (mmHg) & $75 \pm 7,07$ & $91,05 \pm 11,25$ & $98,44 \pm 11,46$ & 0,009 \\
\hline TAD $(\mathrm{mmHg})$ & $45 \pm 7,07$ & $58,16 \pm 7,11$ & $64,53 \pm 9,70$ & 0,005 \\
\hline $\mathrm{CC}(\mathrm{cm})$ & $50 \pm 4,24$ & $59,95 \pm 9,62$ & $68,56 \pm 10,54$ & 0,002 \\
\hline $\operatorname{IMC}\left(\mathrm{Kg} / \mathrm{m}^{2}\right)$ & $13,99 \pm 0,46$ & $17,25 \pm 3,59$ & $19,89 \pm 4,63$ & 0,014 \\
\hline ICT & $0,47 \pm 0,03$ & $0,48 \pm 0,06$ & $0,46 \pm 0,08$ & 0,369 \\
\hline Glucemia Ayunas (mmol/l) & $4,45 \pm 1,06$ & $4,55 \pm 0,87$ & $4,86 \pm 0,84$ & 0,516 \\
\hline Glucemia 2h (mmol/l) & $5,50 \pm 2,26$ & $5,80 \pm 1,58$ & $5,35 \pm 1,16$ & 0,504 \\
\hline Colesterol (mmol/l) & $5,15 \pm 0,35$ & $4,12 \pm 0,75$ & $4,15 \pm 0,91$ & 0,224 \\
\hline $\operatorname{Tg}(\mathrm{mmol} / \mathrm{l})$ & $2,6 \pm 1,70$ & $1,42 \pm 0,58$ & $1,60 \pm 1,54$ & 0,335 \\
\hline $\mathrm{LDL}(\mathrm{mmol} / \mathrm{l})$ & $3,50 \pm 0,14$ & $2,25 \pm 0,74$ & $2,43 \pm 0,70$ & 0,096 \\
\hline Insulinemia & $8,85(2,7)^{*}$ & $10,0026,4)^{*}$ & $9,45(55,8)^{*}$ & 0,759 \\
\hline HOM A-IR & $1,71(0,05)^{*}$ & $2,24(5,21)^{*}$ & $2,21(14,01)^{*}$ & 0,440 \\
\hline
\end{tabular}

\section{Discusión}

En la última década se han ido acumulando evidencias epidemiológicas que relacionan las alteraciones en el eje hormona de crecimiento/factor de crecimiento similar a la Insulina (GH-IGF) y un mayor riesgo de morbilidad cardiovascular ${ }^{15}$. Es ampliamente aceptado que el DGH no tratado conduce a la enfermedad cardiovascular en adultos, por lo que el tratamiento con rhGH en estos produce efectos metabólicos beneficiosos ${ }^{2,16}$. En contraste con lo antes dicho, la situación en la infancia es menos evidente, puesto que en pocos estudios se ha investigado el efecto del DGH y la terapia de reemplazo con rhGH en las alteraciones metabólicas y su asociación con enfermedad cardiovascular. El DGH se presenta con mayor frecuencia en el sexo masculino, nuestro estudio coincide con la mayoría de los estudios que muestran un franco predominio del sexo masculino, $5,7,6,8,17-32$ aunque existen otras investigaciones en los que la muestra está integrada predominantemente por el sexo femenino. ${ }^{29-32}$

$\mathrm{Al}$ evaluar las cifras de la tensión arterial en nuestra investigación señalamos un predominio de la normotensión; es de resaltar que casi la mitad de los pacientes presentan tanto TAS como TAD por debajo del 50 percentil. Los pacientes hipertensos corresponden al sexo masculino y a los mayores de 13 años. Este hallazgo se relaciona con dos factores de riesgo cardiovascular identificados en la infancia, el sexo masculino y la adolescencia. No se encontró variabilidad en este parámetro en cuanto al tiempo de tratamiento, coincidiendo con De Marco et al. ${ }^{29}$ Contrariamente Colao et al., ${ }^{33}$ reportan una disminución significativa tras el inicio del tratamiento. 
Es importante tener en cuenta que las cifras de TA en los niños se incrementan con la edad y la talla corporal, por lo que no existe un punto de corte igual para todos los niños que defina la HTA. De modo que pudiera justificarse porque la talla baja de los niños con DGH, comparadas con la media para la edad, ubica sus cifras de TA en percentiles menores. El IMC ha demostrado ser un indicador apropiado y fácil de determinar para estimar factores de riesgo cardiovascular, al evaluar el estado nutricional de los pacientes. Nuestro estudio reportó que la mitad de los pacientes son normopeso. Esta investigación no encontró diferencias significativas al comparar las medias del ICT según edad y sexo; se identificó una menor prevalencia de los pacientes con obesidad abdominal en aquellos que llevaban más de 5 años de tratamiento, lo cual se justifica porque la hormona de crecimiento ejerce un efecto importante en la distribución de la grasa corporal.

Recientemente Capalbo et al., ${ }^{8}$ en un estudio prospectivo donde se evaluaron 71 nińos con DGH demostró la asociación de esta enfermedad con la presencia de adiposidad abdominal, la cual se redujo tras dos años de terapia de reemplazo con rhGH. Otros estudios ${ }^{30,32}$ reportan valores superiores de circunferencia de cintura y del ICT en nińos con DGH al compararlos con un grupo control. Por otra parte, Ciresi et al. ${ }^{19}$ en un estudio de 105 niños con DGH idiopático señalaron que no existen diferencias significativas con el grupo de control en cuanto la CC. En esta investigación encontramos que la quinta parte de los niños estudiados presentaron alteración del metabolismo de los hidratos de carbono. No encontramos diferencia entre sexos, solo un ligero predominio en las edades superiores. Es de resaltar que fue más frecuente en los niños sin tratamiento con rhGH, mostrándose una disminución de las medias de la glucemia en ayunas y tras la PTG de 2 horas al comparar los grupos según el incremento del tiempo de tratamiento. Witkowska et al. obtuvieron como resultado valores superiores de los niveles de glucemia en ayunas en estos niños. ${ }^{34}$ La mayoría de los estudios ${ }^{4-7,}$ 20-23,25-29, 32, 35-42 donde se compararon los niveles de glucemia en ayunas en niños con DGH sin tratamiento con un grupo control, muestran que no hay diferencias significativas entre estos. En cuanto a las diferencias de sexo, coincidimos con Ciresi et al., ${ }^{19}$ quienes en un estudio retrospectivo evaluaron a 105 niños afectados con DGH, sin encontrar diferencias significativas entre estos; coincidiendo con otros estudios existentes. ${ }^{21,24}$ La mayoría de los estudios han demostrado un incremento en la resistencia a la insulina, debido al aumento de los niveles de insulinemia y el HOMA-IR observado durante la terapia de reemplazo en niños y adolescentes con DGH, a pesar de mantener valores de glucemia en ayunas y postprandiales dentro de rango normal. El incremento de la RI y la disminución de la sensibilidad a la insulina incrementa el riesgo a padecer algún trastorno del metabolismo de los hidratos de carbono y diabetes mellitus. ${ }^{53}$ En el presente estudio vemos la presencia de RI en casi la mitad de los niños, siendo ligeramente superior en el sexo masculino, en las edades mayores y en los niños sin tratamiento con rhGH. Cuando analizamos los valores de insulinemia basal no encontramos diferencias entre sexos, pero el sexo masculino presenta un índice de HOMA-IR superior. Es de señalar que al comparar los valores de las medianas de la insulinemia y el HOMA-IR estas fueron decreciendo paulatinamente en los grupos al incrementarse el tiempo de tratamiento, lo cual se explica por la reducción en los valores de las medias de la CC y el ICT lo que mejora la acción de la insulina a este nivel.

Asimismo, coincidimos con Lanes y et $\mathrm{al}^{43}$, quienes encontraron niveles de insulinemia basales mayores en niños con DGH. Por el contrario, diferimos de las investigaciones revisadas ${ }^{4,6-7,15,20-22,24-27,39,42,44}$ pues al analizar qué ocurre tras iniciar la terapia con rhGH la mayoría de los estudios demostraron un incremento de los niveles de insulinemia. La minoría ${ }^{23}$, 29, 38 no reportó diferencias significativas. 
Al comparar los niveles de insulinemia basal de niños con DGH y nińos sanos la mayoría de los estudios $^{4,7,17,20-23,25-27,29,35,39,42,44}$ reportan que no existen diferencias significativas. Mientras otros ${ }^{24}$, ${ }^{38}$, señalan niveles de insulinemia inferiores en los nińos con DGH. ${ }^{24,34,38}$ El índice HOMA-IR es usado comúnmente en nuestro medio para evaluar la presencia de RI. La mayoría de los estudios ${ }^{4,7,17}$, 20-23, 25-27, 29,35, 39, 42, 44 afirman que la presencia de RI no varía significativamente entre los niños con DGH y los niños sanos. Otros ${ }^{24,30,34}$ muestran niveles de HOMA-IR menores en los niños con DGH. El eje GH/IGF-1 además del papel que juega en el crecimiento lineal promueve un número de efectos metabólicos como la influencia en el metabolismo lipídico. En nuestra investigación encontramos que la tercera parte de estos niños presenta dislipidemia. Dentro de estas predominó la hipertrigliceridemia, identificándose en el tercio de los pacientes. Esta alteración predominó en el sexo femenino siendo estadísticamente significativo. Además, en el sexo femenino los valores de las medias del colesterol, triglicéridos y LDL fueron superiores. Al analizar el comportamiento del perfil lipídico encontramos que las alteraciones de la LDL se presentaron en los menores de 5 ańos y los mayores de 13 años, siendo significativo. Llama la atención este hallazgo, pues durante la adolescencia se produce una disminución fisiológica de los niveles de LDL. Los niños entre 2 y 5 años presentaron valores superiores de colesterol, triglicéridos y LDL comparados con los de mayor edad. La hipertrigliceridemia predominó en los mayores de 13 años, lo que podría corresponder a la elevación fisiológica que ocurre durante la adolescencia. Al comparar estos parámetros en los diferentes grupos según el tiempo en tratamiento con rhGH no encontramos diferencias entre estos; aunque se mostró una tendencia al incremento en el colesterol y la LDL.

Diferimos de los resultados reportados por Ciresi et $\mathrm{al}^{19}$, quienes no encontraron diferencias entre sexos en un estudio retrospectivo donde se evaluaron
105 nińos prepúberes. La mayoría de los estudios revisados ${ }^{33,18,43,45}$ concuerdan en que tras el inicio de la terapia con rhGH se produce una mejoría del perfil lipídico en los pacientes con DGH. En tres estudios recientes, De Marco et al., ${ }^{29} \mathrm{y}$ Capalbo et al., ${ }^{42}$, se reportan niveles significativamente altos de colesterol, triglicéridos y LDL en niños con DGH sin tratamiento. En los cuales, después de 12-24 meses de tratamiento con rhGh el colesterol y la LDL disminuyeron alcanzándose valores similares a los controles. Por su parte, Ramistella et al., ${ }^{4}$ en un estudio prospectivo no encontraron diferencias significativas en el perfil lipídico tras dos años de tratamiento, pero hay un incremento de los triglicéridos y las HDL. Un aumento de las cifras de triglicéridos y disminución de las LDL tras un año de tratamiento fue notificado por Witkouska-Sedek et al. ${ }^{34}$ Algunos estudios no encontraron cambios significativos en el perfil lipídico después de 12 meses de tratamiento. , $17,36,46$ La mayoría de los estu$\operatorname{dios}^{8,18,28,37,47,43}$ al comparar los niños con DGH con niños sanos señalan un perfil lipídico desfavorable en los primeros. En algunas investigaciones ${ }^{17,36}$ no se detectan diferencias. Sin embargo, De Marco et al. ${ }^{29}$ señalan cifras de triglicéridos inferiores en los niños con DGH.

\section{Conclusiones}

La DGH puede tener un efecto negativo en la composición corporal y el estado metabólico. El tratamiento con rhGH en niños conlleva una composición corporal más favorable con menor porcentaje de masa grasa y mayor de masa magra. Tiene un efecto positivo sobre el perfil lipídico, ya que se observa una reducción significativa en el índice aterogénico. En este estudio predominó la hipertrigliceridemia en el sexo femenino, mientras las elevaciones de las LDL fueron más frecuentes en los menores de cinco años. Los valores de tensión arterial (TA), circunferencia de la cintura (CC) y el índice de masa corporal (IMC) se incrementan de forma significativa con la edad. En los nińos predominaron las alteraciones corporales, 
mientras que en las niñas fueron las alteraciones del lipidograma.

\section{Bibliografia}

1. Cañete R, Gil M. Déficit de hormona de crecimiento. En Pombo A. Tratado de Endocrinología Pediátrica, McGraw-Hill Interamericana de España. Madrid. 2009; 23:284-98.

2. Isgaard J, Arcopinto M, Karason K, Cittadini A. $\mathrm{GH}$ and the cardiovascular system: an update on a topic at heart. Endocrine. 2015;48(1):25-35.

3. De Leonibus C, De Marco S, Stevens A, Clayton P, Chiarelli F, Monh A. Growth hormone deficiency in prepubertal children: predictive markers of cardiovascular disease. Horm Res Paediatr. 2016;85(6):363-71.

4. Ramistella V, Wasniewska M, Arasi S, Catena M, Velletri MR, Corica D, et al. Cross-sectional and prospective study of the effects of $\mathrm{GH}$ therapy on metabolic panel in children with GH deficiency. Pediatr Med Chir. 2014;36(5):104. Available from https://doi.org/10.4081/pmc.2014.104

5. Ciresi A, Ciccio F, Amato MC, Giordano C. Revaluation of the clinical and metabolic behavior of children with isolated growth hormone deficiency during GH treatment according to newly proposed note 39 of the Italian Medicines Agency (AIFA). J Endocrinol Investig. 2015;38(12):1301-7.

6. Meazza C, Elsedfy HH, Pagani S, Bozzola E, El Kholy M, Bozzola M. Metabolic parameters and adipokine profile in growth hormone deficient (GHD) children before and after 12-month GH treatment. Horm Metab Res. 2014;46(3):219-23.

7. Metwalley KA, Farghaly HS, Abd El-Hafeez HA. Evaluation of left ventricular mass and function, lipid profile, and insulin resistance in Egyptian children with growth hormone deficiency: a single-center prospective case-control study. Indian J EndocrinolMetab. 2013;17 (5):876-82.

8. Capalbo D, Mattace RG, Esposito A, Di Mase $\mathrm{R}$, Barbieri F, Meli R, et al. Cluster of cardiometabolic risk factors in children with $\mathrm{GH}$ deficiency: a prospective, case-control study. Clin Endocrinol (Oxf) 2014;80(6):856-62.

9. Labarta J, De Arriba A, Ferrer M, Ruiz I, López M, Lou G. Mesa redonda: Hormona de crecimiento y riesgo cardiovascular. Rev Esp Endocrinol Pediatr 2016;7:24-9.

10. Hernández J, Duchi PN. Índice cintura/talla y su utilidad para detectar riesgo cardiovascular y metabólico. Rev Cubana Endocrinol. 2015;26(1):66-76.

11. Guía cubana de diagnóstico, evaluación y tratamiento de la hipertensión arterial. La Habana: Revista Cubana de Medicina. 2017;56:49-53.

12. Instituto Nacional de Endocrinología. Manual de Diagnóstico y Tratamiento en Endocrinología y Metabolismo. La Habana, 2015. (En prensa).

13. Cabrera E, Marichal S, Parlá J, Arrans C, González R, Pérez C, et al. Frecuencia y características del síndrome metabólico y de la resistencia a la insulina en familiares de primer grado de personas con diabetes mellitus tipo 1. Endocrinol Nutr. 2010;57(7):311-21.

14. Jellinger PS, Handelsman Y, Rosenblit PD, Bloomgarden ZT, Fonseca VA, Garber AJ, et al. American Association of Clinical Endocrinologists and American College of Endocrinology Guidelines for Management of Dyslipidemia and Prevention of Atherosclerosis. Endocr Pract. 2017;23(4):479-97. 
15. Khadilkar V, Ekbote V, Kajale N, Khadilkar A, Chiplonkar S, Kinare A. Effect of one year growth hormone therapy on body composition and cardio-metabolic risk in Indian children with growth hormone deficiency, Endocr. Res. 2014;39:73-8.

16. Gazzaruso C, Gola M, Karamouzis I, Giubbini R, Giustina A. Cardiovascular risk in adult patients with growth hormone (GH) deficiency and following substitution with $\mathrm{GH}$ an update. J Clin Endocrinol Metab. 2014;99:18-29.

17. Ciresi A, Cicciò F, Radellini S, Giordano C. Utility of C-peptide for a reliable estimate of insulin secretion in children with growth hormone deficiency. Growth Horm IGF Res. 2016;29:71-7.

18. Abbas K, Saad H, Ahmed H. Evaluation of left ventricular mass and function, lipid profile and insulin resistanceinEgyptianchildrenwithgrowth hormone deficiency: A single case-control study. Indian J Endocrinol Metab.2013;17(5):876-82. Doi: $10.4103 / 2230-8210.117234$

19. Ciresi A, Radellini S, Guarnotta V, Mineo MG, Giordano C. Los resultados metabólicos del tratamiento con hormona de crecimiento en niños son específicos de género. Endocr Connect. 2018;7(7):879-87. Doi: 10.1530 / EC-18-0135

20. Seminara S, Merello G, Masi S, Filpo A, La Cauza F, D'Onghia G, et al. Effect of long-term growth hormone treatment on carbohydrate metabolism in children with growth hormone deficiency. Clin Endocrinol. 1998;49(1):125-30.

21. Salerno M, Esposito V, Farina V, Radetti G, Umbaldo A, Capalbo D, et al. Improvement of cardiac performance and cardiovascular risk factors in children with GH deficiency after two years of GH replacement therapy: an observa- tional, open, prospective, case-control study. J Clin Endocrinol Metab. 2006;91(4):1288-95.

22. Ciresi A, Amato MC, Criscimanna A, Mattina A, Vetro C, Galluzzo A, et al. Metabolic parameters and adipokine profile during GH replacement therapy in children with GH deficiency. Eur J Endocrinol. 2007;156(3):353-60.

23. López-Siguero JP, López-Canti LF, Espino R, Caro E, Fernández-García JM, Gutiérrez-Macías A, et al. Effect of recombinant growth hormone on leptin, adiponectin, resistin, interleukin- 6 , tumor necrosis factor- $\alpha$ and ghrelin levels in growth hormone-deficient children. J Endocrinol Invest. 2011;34(4):300-6.

24. Cañete R, Valle M, Martos R, Sánchez-Carrión A, Cańete MD, van Donkelaar E. Shortterm effects of GH treatment on coagulation, fibrinolysis, inflammation biomarkers, and insulin resistance status in prepubertal children with GH deficiency. Eur J Endocrinol. 2012; 167(2):255-60.

25. Ciresi A, Giordano C. One-hour post-load plasma glucose level is associated with a worse metabolic profile in children with GH deficiency. J Endocrinol Invest. 2018;41:789-97.

26. Ciresi A, Guarnotta V, Pizzolanti G, Giordano C. Comparison between euglycemic hyperinsulinemic clamp and surrogate indices of insulin sensitivity in children with growth hormone deficiency. Growth Horm IGF Res. 2017;39:40-4.

27. Ciresi A, Pizzolanti G, Leotta M, Guarnotta V, Teresi G, Giordano C. Resistin, visfatin, leptin and omentin are differently related to hormonal and metabolic parameters in growth hormone-deficient children. J Endocrinol Inves.2016; 39(9):1023-30. 
28. Stawerska R, Smyczyńska J, Hilczer M, Lewiński A. Relationship between IGF-I concentration and metabolic profile in children with growth hormone deficiency: the influence of children's nutritional state as well as the ghrelin, leptin, adiponectin, and resistin serum concentrations. Int J Endocrinol. 2017; 4:1-9.

29. De Marco S, Marcovecchio ML, Caniglia D, De Leonibus C, Chiarelli F, Mohn A. Circulating asymmetric dimethylarginine and lipid profile in pre-pubertal children with growth hormone deficiency: effect of 12-month growth hormone replacement therapy. Growth Horm IGF Res. 2014;24(5):216-20.

30. Xie X, Li Q, Zhang L, Ren W. Lipid accumutation product, visceral adiposity index, and Chinese visceral adiposity index as markers of cardiometabolic risk in adult growth hormone deficiency patients: a cross-sectional study. Endocrine practice. 2018;24(1):33-9.

31. Uzunova I, Kirilov G, Zacharieva S, Shinkov A, Borissova AM, Kalinov K, et al. Individual risk factors of the metabolic syndrome in adult patients with growth hormona deficiency: A cross-sectional case-control study. Exp Clin Endocrinol Diabetes. 2015;123:39-43.

32. Chan L, Xue H, Xiaoya Z, Jiajia X, Wei R,Linman L, et al. Lipid accumulation product: a simple and accurate index for predicting Metabolic Syndrome in patients with adult growth hormone deficiency. Exp Clin Endocrinol Diabetes. 2016;124(4):220-4. Doi:10.1055/ s-0035-1569402.

33. Colao A, Di Somma, Salerno M, Pivonello R, Cuocolo A, SpinelliL, et al. The cardiovascular risk of GH-deficient adolescents. J Clin Endocrinol Metab. 2002;87(8):3650-5.
34. Witkowska-Sedek E, Rumisnska M, Stelmazezyk-Enmel A, Majcher A, Pyrzak B. The associations between the growth hormone/ insulin like growth factor-1 axis, adiponectin, resistin and metabolic profile in children with growth hormone deficiency before and during growth hormone treatment. Acta biochimica polonice. 2018;65(2):333-40.

35. Prodam F, SavastioS, Genoni G, Babu D, Giordano M, Ricotti R, et al. Effects of growth hormone $(\mathrm{GH})$ therapy withdrawal on glucose metabolism in not confirmed GH deficient adolescents at final height. PLoS One. 2014;9(1):857-71.

36. Liang S, Xue J, Li G. Effects of recombinant human growth hormone administration on cardiovascular risk factors in obese children with relative growth hormone deficiency. Lipids Health Dis. 2018;17:66. Doi: 10.1186/s12944018-0721-9

37. Chen M, Gan D, Luo Y, Rampersad S, Xu Y, Yang $S$, et al. Effect of recombinant human growth hormone therapy on blood lipid and carotid intima-media thickness in children with growth hormone deficiency. Pediatr Res. 2018;83(5):954-60.

38. Lippe BM, Kaplan SA, Golden MP, Hendricks SA, Scott ML. Carbohydrate tolerance and insulin receptor binding in children with hypopituitarism: response after acute and chronic human growth hormone administration. J Clin Endocrinol Metab. 1981;53(3):507-13.

39. Heptulla RA, Boulware SD, Caprio S, Silver D, Sherwin RS, Tamborlane WV. Decreased insulin sensitivity and compensatory hyperinsulinemia after hormone treatment in children with short stature. J Clin Endocrinol Metab. 1997;82(10):3234-8. 
40. Xue Y, Gao Y, Wang S, Wang P. An examination of the effects of different doses of recombinant human growth hormone on children with growth hormone deficiency. Exp Ther Med. 2016;11(5):1647-52.

41. Costa U, Oliveira C, Salvatori R, Barreto-Filho JA, Campos V, Oliveira F, et al. Brazilian adult individuals with untreated isolated GH deficiency do not have acceleratted subclinical atherosclerosis. Endocr connect. 2016;5(1):41-6.

42. Capalbo D, Esposito A, Improda N, Wasniewska MG, Di Mase R, De Luca F, et al. Glucose homeostasis in GHD children during long-term replacement therapy: a case-control study. Endocrine. 2018;59(3):643-50.

43. Lanes R. Cardiovascular risk in growth hormone deficiency. Beneficial effects of growth hormone replacement therapy. Endocrinol Metab Clin N Am. 2016;45:405-18.
44. Ciresi A, Amato MC, Giordano C. Reduction in insulin sensitivity and inadequate $\beta$-cell capacity to counteract the increase in insulin resistance in children with idiopathic growth hormone deficiency during 12 months of growth hormone treatment. J Endocrinol Invest.2015;38(3):351-9. Doi: 10.1007/s40618-014-0184-4.

45. Kubo T, Furujo M, Takahashi K, Hyodo Y, Tsuchiya H, Hattori M, et al. Effects of Growth Hormone Treatment on Lipid Profiles. Indian J Pediatr. 2017;1-5. Available from https://doi. org/10.1007/s12098-017-2509-8

46. Marcosyan R, Perikhanyan A. Recombinant growth hormone therapy in children with GH deficiency: first interventional study in Armenia. Value in Health.2014;17:336.

47. Binay C, Simsek E, Yildirim A, Kosger P, Demiral M, Kilic Z.Growth hormone and the risk of atherosclerosis in growth hormone deficient children. Growth Horm IGF Res. 2015;25(6):294-7. 